\title{
A numerical study of microparticle acoustophoresis driven by acoustic radiation forces and streaming-induced drag forces
}

Muller, Peter Barkholt; Barnkob, Rune; Jensen, Mads Jakob Herring; Bruus, Henrik

Published in:

Lab on a Chip

Link to article, DOI:

10.1039/c2lc40612h

Publication date:

2012

Document Version

Publisher's PDF, also known as Version of record

Link back to DTU Orbit

Citation (APA):

Muller, P. B., Barnkob, R., Jensen, M. J. H., \& Bruus, H. (2012). A numerical study of microparticle acoustophoresis driven by acoustic radiation forces and streaming-induced drag forces. Lab on a Chip, 12(22), 4617-4627. https://doi.org/10.1039/c2lc40612h

\section{General rights}

Copyright and moral rights for the publications made accessible in the public portal are retained by the authors and/or other copyright owners and it is a condition of accessing publications that users recognise and abide by the legal requirements associated with these rights.

- Users may download and print one copy of any publication from the public portal for the purpose of private study or research.

- You may not further distribute the material or use it for any profit-making activity or commercial gain

- You may freely distribute the URL identifying the publication in the public portal 


\title{
A numerical study of microparticle acoustophoresis driven by acoustic radiation forces and streaming-induced drag forces
}

\author{
Peter Barkholt Muller, ${ }^{a}$ Rune Barnkob, ${ }^{b}$ Mads Jakob Herring Jensen ${ }^{c}$ and Henrik Bruus*a
}

Received 28th May 2012, Accepted 18th July 2012

DOI: $10.1039 / \mathrm{c} 2 \mathrm{lc40612h}$

\begin{abstract}
We present a numerical study of the transient acoustophoretic motion of microparticles suspended in a liquid-filled microchannel and driven by the acoustic forces arising from an imposed standing ultrasound wave: the acoustic radiation force from the scattering of sound waves on the particles and the Stokes drag force from the induced acoustic streaming flow. These forces are calculated numerically in two steps. First, the thermoacoustic equations are solved to first order in the imposed ultrasound field taking into account the micrometer-thin but crucial thermoviscous boundary layer near the rigid walls. Second, the products of the resulting first-order fields are used as source terms in the time-averaged second-order equations, from which the net acoustic forces acting on the particles are determined. The resulting acoustophoretic particle velocities are quantified for experimentally relevant parameters using a numerical particle-tracking scheme. The model shows the transition in the acoustophoretic particle motion from being dominated by streaming-induced drag to being dominated by radiation forces as a function of particle size, channel geometry, and material properties.
\end{abstract}

\section{Introduction}

In the past decade there has been a markedly increasing interest in applying ultrasound acoustofluidics as a tool for purely mechanical and label-free manipulation of particle and cell suspensions in MEMS and biologically oriented lab-on-a-chip systems. Recent extended reviews of acoustofluidics can be found in Review of Modern Physics ${ }^{1}$ and the tutorial series in Lab on a Chip ${ }^{2}$ which, among other topics, treats the application of ultrasound bulk ${ }^{3}$ and surface ${ }^{4}$ acoustic waves as well as acoustic forces on particles from acoustic radiation ${ }^{5}$ and from streaminginduced drag. ${ }^{6}$

When a standing ultrasound wave is established in a microchannel containing a microparticle suspension, the particles are subject to two acoustic forces: the acoustic radiation force from the scattering of sound waves on the particles, and the Stokes drag force from the induced acoustic streaming flow. The resulting motion of a given particle is termed acoustophoresis, migration by sound. Experimental work on acoustophoresis has mainly dealt with the radiation force, primarily because this force dominates over the streaming-induced drag force for the studied aqueous suspensions of polymer particles or biological cells with diameters larger than $2 \mu \mathrm{m}$. Detailed measurements of

${ }^{a}$ Department of Physics, Technical University of Denmark, DTU Physics Building 309, DK-2800 Kongens Lyngby, Denmark.

E-mail:bruus@fysik.dtu.dk

${ }^{b}$ Department of Micro- and Nanotechnology, Technical University of Denmark, DTU Nanotech Building 345 East, DK-2800 Kongens Lyngby, Denmark

${ }^{c}$ COMSOL A/S, Diplomvej 373, DK-2800 Kongens Lyngby, Denmark the acoustophoretic motion of large $5 \mu \mathrm{m}$ diameter polystyrene particles in water ${ }^{7,8}$ have shown good agreement with the theoretical predictions ${ }^{9,10}$ for the radiation force on compressible particles with a radius $a$ much smaller than the acoustic wavelength $\lambda$ and neglecting the viscosity of the suspending fluid.

However, as the particle diameter $2 a$ is reduced below $2 \mu \mathrm{m}$, viscous effects are expected to become significant, because this length corresponds to a few times the viscous penetration depth or boundary-layer thickness $\delta$. Analytical expressions for the viscous corrections to the radiation force valid in the experimentally relevant limit of long wavelength $\lambda$, characterized by $a$ $\ll \lambda$ and $\delta \ll \lambda$, have been given recently, ${ }^{11}$ but have not yet been tested experimentally. In addition to these modifications of the radiation force, the acoustic streaming flow induced by viscous stresses in the boundary layers near rigid walls, and depending critically on the detailed geometry and boundary conditions, also significantly influences the acoustophoretic particle motion as the size of the particle or the confining microchannel is reduced. ${ }^{12,13}$ The cross-over from radiation-dominated to streaming-dominated motion has been observed in experiments, ${ }^{14,15}$ and a scaling analysis of the critical particle diameter for this cross-over has been provided in the literature ${ }^{16}$ and will be restated in Section IV D.

Although acoustic streaming is a well-known phenomenon in acoustics, it is pointed out in a recent review ${ }^{6}$ that streaming is often misunderstood outside the relatively small circles of acoustics experts due to the many forms in which it may arise in, e.g., acoustofluidic microsystems. Not only is acoustic streaming difficult to predict quantitatively due to its sensitivity 
to the detailed geometry and boundary conditions, but part of the more conceptual difficulties with acoustic streaming in acoustofluidics may be related to the lack of theoretical analysis in the experimental relevant limit where the microchannel height $h$ is equal to one or a few times half the acoustic wavelength $\lambda$, i.e. $h \simeq \lambda$. The classical Rayleigh-Schlichting boundary-layer theory for acoustic streaming, ${ }^{17-20}$ see Fig. 1, is valid in the limit of thin boundary layers in medium-sized channels, $\delta \ll h \ll \lambda$, and a later extension ${ }^{13}$ is valid in the limit of thin boundary layers in shallow channels, $\delta \simeq h \ll \lambda$. Moreover, in contrast to rectangular channel cross sections of experimental relevance, the classical analysis of the parallel-plate channel and recent numerical studies of $i^{21}$ do not include the effects of the vertical side walls. One exception is the special case of gases in shallow, low-aspect-ratio channels studied by Aktas and Farouk. ${ }^{22}$

The push within contemporary acoustofluidics for handling smaller particles like bacteria, viruses, and large biomolecules, and for doing so with better accuracy, emphasizes the urgency of performing a numerical analysis of microparticle acoustophoresis including acoustic radiation forces, streaming flows, and boundary layers. Based directly on the governing equations, we provide such an analysis in this paper for a simple, yet experimentally relevant microsystem. In Section II we present the governing thermoacoustic equations to first and second order in the external ultrasound actuation. In Section III we describe the model system, the numerical implementation of it, as well as mesh-convergence analysis. In Section IV this is followed by the results for first-order fields, time-averaged second-order fields, and microparticle acoustophoresis as function of particle size and material properties. We end with a concluding discussion in Section V.

\section{Governing equations}

The governing perturbation equations for the thermoacoustic fields are well-known textbook material. ${ }^{23-25}$ The full acoustic

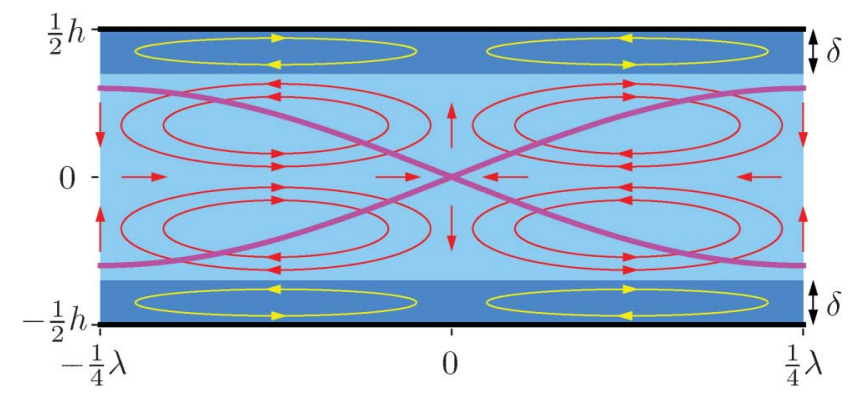

Fig. 1 A sketch of the classical Rayleigh-Schlichting streaming pattern in a liquid-filled gap of height $h$ between two infinite, parallel rigid walls (black lines). The bulk liquid (light blue) supports a horizontal standing sinusoidal pressure wave (magenta line) of wavelength $\lambda$ in the horizontal direction parallel to the walls. In the viscous boundary layers (dark blue) of sub-micrometer thickness $\delta$, large shear stresses appear, which generate the boundary-layer (Schlichting) streaming rolls (yellow). These then drive the bulk (Rayleigh) streaming rolls (red). The streaming pattern is periodic in the horizontal direction with periodicity $\lambda / 2$, and thus only the top and bottom walls are subject to the no-slip boundary condition. problem in a fluid, which before the presence of any acoustic wave is quiescent with constant temperature $T_{0}$, density $\rho_{0}$, and pressure $p_{0}$, is described by the four scalar fields pressure $p$, temperature $T$, density $\rho$, and entropy $s$ per mass unit as well as the velocity vector field $\boldsymbol{v}$. Changes in $\rho$ and $s$ are given by the two thermodynamic relations

$$
\begin{aligned}
\mathrm{d} \rho & =\gamma \kappa \rho \mathrm{d} p-\alpha \rho \mathrm{d} T, \\
\mathrm{~d} s & =\frac{C_{p}}{T} \mathrm{~d} T-\frac{\alpha}{\rho} \mathrm{d} p,
\end{aligned}
$$

which, besides the specific heat capacity $C_{p}$ at constant pressure, also contain the specific heat capacity ratio $\gamma$, the isentropic compressibility $\kappa$, and the isobaric thermal expansion coefficient $\alpha$ given by

$$
\begin{aligned}
& \gamma=\frac{C_{p}}{C_{V}}, \\
& \kappa=\frac{1}{\rho}\left(\frac{\partial \rho}{\partial p}\right)_{s} \\
& \alpha=-\frac{1}{\rho}\left(\frac{\partial \rho}{\partial T}\right)_{p} .
\end{aligned}
$$

Eqn (1) can be used to eliminate $\rho$ and $s$, so that we only need to deal with the acoustic perturbations in temperature $T$, pressure $p$, and velocity $\boldsymbol{v}$. Taking first and second order (subscript 1 and 2, respectively) into account, we write the perturbation series

$$
\begin{aligned}
& T=T_{0}+T_{1}+T_{2}, \\
& p=p_{0}+p_{1}+p_{2}, \\
& v=v_{1}+v_{2} .
\end{aligned}
$$

We model the external ultrasound actuation through boundary conditions on the first-order velocity $\boldsymbol{v}_{1}$ while keeping the temperature constant,

$$
\begin{aligned}
T & =T_{0}, \text { on all walls, } \\
\boldsymbol{v} & =\mathbf{0}, \text { on all walls, } \\
\boldsymbol{n} \cdot \boldsymbol{v}_{1} & =v_{\mathrm{bc}}(y, z) \mathrm{e}^{-\mathrm{i} \omega t}, \text { added to actuated walls. }
\end{aligned}
$$

Here $\boldsymbol{n}$ is the outward pointing surface normal vector, and $\omega$ is the angular frequency characterizing the harmonic time dependence.

\section{A First-order equations}

To first order in the amplitude of the imposed ultrasound field, the thermodynamic heat transfer equation for $T_{1}$, the kinematic continuity equation expressed in terms of $p_{1}$, and the dynamic Navier-Stokes equation for $\boldsymbol{v}_{1}$, become

$$
\partial_{t} T_{1}=D_{\mathrm{th}} \nabla^{2} T_{1}+\frac{\alpha T_{0}}{\rho_{0} C_{p}} \partial_{t} p_{1},
$$




$$
\begin{aligned}
\partial_{t} p_{1} & =\frac{1}{\gamma \kappa}\left[\alpha \partial_{t} T_{1}-\nabla \cdot \boldsymbol{v}_{1}\right], \\
\rho_{0} \partial_{t} \boldsymbol{v}_{1} & =-\nabla p_{1}+\eta \nabla^{2} \boldsymbol{v}_{1}+\beta \eta \nabla\left(\nabla \cdot \boldsymbol{v}_{1}\right) .
\end{aligned}
$$

Here, $D_{\text {th }}$ is the thermal diffusivity, $\eta$ is the dynamic viscosity, and $\beta$ is the viscosity ratio, which has the value $1 / 3$ for simple liquids. ${ }^{23} \mathrm{~A}$ further simplification can be obtained when assuming that all first-order fields have a harmonic time dependence $\mathrm{e}^{-\mathrm{i} \omega t}$ inherited from the imposed ultrasound field (eqn (4c)), because then $p_{1}$ is eliminated by inserting eqn (5b) with $\partial_{t} p_{1}=-\mathrm{i} \omega p_{1}$ into eqn (5a) and (c). After using the thermodynamic identity ${ }^{26} T_{0} \alpha^{2} /\left(\rho_{0} C_{p} \kappa\right)=\gamma-1$, we arrive at

$$
\begin{array}{r}
\mathrm{i} \omega T_{1}+\gamma D_{\mathrm{th}} \nabla^{2} T_{1}=\frac{\gamma-1}{\alpha} \nabla \cdot \boldsymbol{v}_{1}, \\
\mathrm{i} \omega \boldsymbol{v}_{1}+v \nabla^{2} \boldsymbol{v}_{1}+v\left[\beta+\mathrm{i} \frac{1}{\gamma \rho_{0} \kappa v \omega}\right] \nabla\left(\nabla \cdot \boldsymbol{v}_{1}\right)=\frac{\alpha}{\gamma \rho_{0} \kappa} \nabla T_{1},
\end{array}
$$

where $v=\eta / \rho_{0}$. From eqn (6) arise the thermal and the viscous penetration depth $\delta_{\text {th }}$ and $\delta$, respectively (values for ultrasound waves at $2 \mathrm{MHz}$ in water at $25^{\circ} \mathrm{C}$ ),

$$
\delta_{\text {th }}=\sqrt{\frac{2 D_{\text {th }}}{\omega}}=0.15 \mu \mathrm{m}, \quad \text { and } \quad \delta=\sqrt{\frac{2 v}{\omega}}=0.38 \mu \mathrm{m} .
$$

These are the length scales over which the thermoacoustic fields change from their bulk values to the boundary conditions of the rigid walls stated in eqn (4).

\section{B Second-order, time-averaged equations}

For water and most other liquids, the thermal effects in the above first-order equations are minute because of the smallness of the pre-factor $\gamma-1 \approx 10^{-2}$ and $\delta_{\mathrm{th}} / \delta \approx 0.3$. To simplify the following treatment, we therefore neglect the coupling in the second-order equations between the temperature field $T_{2}$ and the mechanical variables $v_{2}$ and $p_{2}$. Furthermore, the values of $\eta$ and $\beta$ are kept fixed at the ones given at $T=T_{0}$. The secondorder continuity equation and Navier-Stokes equation are

$$
\begin{aligned}
\partial_{t} \rho_{2}= & -\rho_{0} \nabla \cdot \boldsymbol{v}_{2}-\nabla \cdot\left(\rho_{1} \boldsymbol{v}_{1}\right), \\
\rho_{0} \partial_{t} \boldsymbol{v}_{2}= & -\nabla p_{2}+\eta \nabla^{2} \boldsymbol{v}_{2}+\beta \eta \nabla\left(\nabla \cdot \boldsymbol{v}_{2}\right) \\
& -\rho_{1} \partial_{t} \boldsymbol{v}_{1}-\rho_{0}\left(\boldsymbol{v}_{1} \cdot \nabla\right) \boldsymbol{v}_{1},
\end{aligned}
$$

and consequently, thermal effects enter solely through the temperature-dependent first-order fields $\rho_{1}$ and $\boldsymbol{v}_{1}$.

In a typical experiment on microparticle acoustophoresis, the microsecond timescale of the ultrasound oscillations is not resolved. It therefore suffices to treat only the time-averaged equations. The time average over a full oscillation period, denoted by the angled brackets $\langle\ldots\rangle$, of the second-order continuity equation and Navier-Stokes equation becomes ${ }^{27}$

$$
\rho_{0} \nabla \cdot\left\langle\boldsymbol{v}_{2}\right\rangle=-\nabla \cdot\left\langle\rho_{1} \boldsymbol{v}_{1}\right\rangle,
$$

$$
\begin{gathered}
\eta \nabla^{2}\left\langle\boldsymbol{v}_{2}\right\rangle+\beta \eta \nabla\left(\nabla \cdot\left\langle\boldsymbol{v}_{2}\right\rangle\right)-\left\langle\nabla p_{2}\right\rangle \\
=\left\langle\rho_{1} \partial_{t} \boldsymbol{v}_{1}\right\rangle+\rho_{0}\left\langle\left(\boldsymbol{v}_{1} \cdot \nabla\right) \boldsymbol{v}_{1}\right\rangle .
\end{gathered}
$$

It is seen that products of first-order fields act as source terms (at the right-hand sides) for the second-order fields (at the left-hand sides). We note that for complex-valued fields $A(t)$ and $B(t)$ with harmonic time-dependence $\mathrm{e}_{1}^{-\mathrm{i} \omega t}$, the time average is given by the real-part rule $\langle A(t) B(t)\rangle=\frac{1}{2} \operatorname{Re}\left[A(0)^{*} B(0)\right]$, where the asterisk represents complex conjugation.

The second-order problem was solved in the case of the infinite parallel-plate channel by Rayleigh, ${ }^{17,20}$ see Fig. 1. Assuming a first-order bulk velocity field with only the horizontal $y$-component $v_{1 y}$ being non-zero and of the form $v_{1 y}$ $=U_{1} \cos (2 \pi y / \lambda) \mathrm{e}^{-\mathrm{i} \omega t}$, the resulting $y$-component $\left\langle v_{2 y}^{\text {bnd }}\right\rangle$ of $\left\langle\boldsymbol{v}_{2}\right\rangle$ just outside the boundary layers (in our notation at $z \approx \pm[h / 2-$ $3 \delta]$ ), becomes

$$
\left\langle v_{2 y}^{\text {bnd }}\right\rangle=\frac{3}{8} \frac{U_{1}^{2}}{c_{0}} \sin \left(4 \pi \frac{y}{\lambda}\right)
$$

Relative to the first-order bulk velocity $v_{1 y}$, the second-order field $\left\langle v_{2 y}^{\text {bnd }}\right\rangle$ is phase shifted by $\pi / 2$, period doubled in space, and smaller by a factor of $U_{1} / c_{0}$, where $c_{0}$ is the speed of sound of the liquid.

\section{Time-averaged acoustic forces on a single suspended microparticle}

Once the first- and second-order acoustic fields have been calculated, it is possible to determine the time-averaged acoustic forces on a single suspended particle. These are the acoustic radiation force $\boldsymbol{F}^{\text {rad }}$ due to the scattering of acoustic waves on the particle and the Stokes drag force $\boldsymbol{F}^{\mathrm{drag}}$ from the acoustic streaming.

The time-average acoustic radiation force $\boldsymbol{F}^{\mathrm{rad}}$ on a single small spherical particle of radius $a$, density $\rho_{\mathrm{p}}$, and compressibility $\kappa_{\mathrm{p}}$ in a viscous fluid is given by ${ }^{11}$

$$
\boldsymbol{F}^{\mathrm{rad}}=-\pi a^{3}\left[\frac{2 \kappa_{0}}{3} \operatorname{Re}\left[f_{1}{ }^{*} p_{1}{ }^{*} \nabla p_{1}\right]-\rho_{0} \operatorname{Re}\left[f_{2}{ }^{*} \boldsymbol{v}_{1}{ }^{*} \cdot \nabla \boldsymbol{v}_{1}\right]\right],
$$

where $\kappa_{0}=1 /\left(\rho_{0} c_{0}^{2}\right)$ is the compressibility of the fluid, and where the pre-factors $f_{1}$ and $f_{2}$ are given by

$$
\begin{array}{cc}
f_{1}(\tilde{\kappa})=1-\tilde{\kappa}, & \text { with } \tilde{\kappa}=\frac{\kappa_{\mathrm{p}}}{\kappa_{0}}, \\
f_{2}(\tilde{\rho}, \tilde{\delta})=\frac{2[1-\Gamma(\tilde{\delta})](\tilde{\rho}-1)}{2 \tilde{\rho}+1-3 \Gamma(\tilde{\delta})}, & \text { with } \tilde{\rho}=\frac{\rho_{\mathrm{p}}}{\rho_{0}}, \\
\Gamma(\tilde{\delta})=-\frac{3}{2}[1+\mathrm{i}(1+\tilde{\delta})] \tilde{\delta}, & \text { with } \tilde{\delta}=\frac{\delta}{a},
\end{array}
$$

For the special case of the horizontal pressure half-wave resonance, $p_{1}=p_{\mathrm{a}} \sin (q y)$, with channel width $w$ and wavenumber $q=\pi / w$, the acoustic energy density is $E_{\mathrm{ac}}=\frac{1}{4} \kappa_{0} p_{\mathrm{a}}^{2}=\frac{1}{4} \rho_{0} U_{1}^{2}$. The expression for the radiation force then simplifies to 


$$
\begin{aligned}
F_{1 \mathrm{D}}^{\mathrm{rad}} & =4 \pi \Phi(\tilde{\kappa}, \tilde{\rho}, \tilde{\delta}) a^{3} q E_{\mathrm{ac}} \sin (2 q y), \\
\Phi(\tilde{\kappa}, \tilde{\rho}, \tilde{\delta}) & =\frac{1}{3} f_{1}(\tilde{\kappa})+\frac{1}{2} \operatorname{Re}\left[f_{2}(\tilde{\rho}, \tilde{\delta})\right],
\end{aligned}
$$

where $\Phi$ is the so-called acoustic contrast factor.

The time-averaged Stokes drag force $\boldsymbol{F}^{\text {drag }}$ on a spherical particle of radius $a$ moving with velocity $\boldsymbol{u}$ in a fluid having the streaming velocity $\left\langle\boldsymbol{v}_{2}\right\rangle$ is given by the usual expression

$$
\boldsymbol{F}^{\mathrm{drag}}=6 \pi \eta a\left(\left\langle\boldsymbol{v}_{2}\right\rangle-\boldsymbol{u}\right),
$$

valid for particles sufficiently far from the channel walls. ${ }^{28}$

\section{Numerical model}

In the following we present the idealized numerical model, and how we implement and solve the governing equations for this model using the finite element software COMSOL Multiphysics 4.2a, see ref. 29.

\section{A Model system and computational domain}

Given the detailed measurements of the acoustophoretic motion and the successful comparison with theoretical predictions presented in ref. 7 and 8 , it is natural to use an idealization of their straight microchannel of length $35 \mathrm{~mm}$ and rectangular cross section as a model system in our numerical study. We neglect the chip structure and simply represent the silicon-glass chip as hardwall boundary conditions. We further neglect any axial dynamics in the long straight channel, and thus restrict our analysis to the rectangular cross section $\Omega$ of width $w=0.38 \mathrm{~mm}$ and height $h=$ $0.16 \mathrm{~mm}$ in the vertical $y z$-plane, see Fig. 2. Finally, we represent the ultrasonic piezo transducer by the velocity boundary condition eqn (4). The particle suspensions are modeled as being monodisperse and containing non-interacting, spherical polystyrene particles with diameters of $0.5,1.0,2.0,3.0$, or $5.0 \mu \mathrm{m}$, respectively.

The model system has a horizontal half-wave resonance across the width $w$ given by the frequency $f=\omega /(2 \pi)=c_{0} /(2 w)$, equal to 1.97 $\mathrm{MHz}$ for water. To excite this resonance, we let all external acoustic excitations have a harmonic time dependence of frequency $f=1.97 \mathrm{MHz}$. All relevant material parameters are listed in Table 1 .

\section{B Particle tracing model}

In order to study the acoustophoretic motion of $N$ particles suspended in the microchannel, we apply the COMSOL Particle Tracing Module, which closely mimics experimental particle
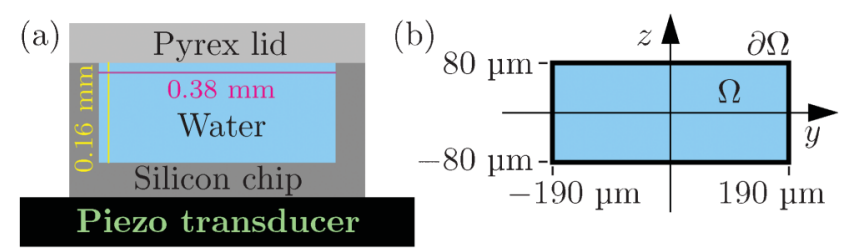

Fig. 2 (a) End-view sketch of the acoustophoresis microchip with a fluidic channel of width $w=0.38 \mathrm{~mm}$ and height $h=0.16 \mathrm{~mm}$ used in experiments. ${ }^{7,8}$ It consists of a silicon chip (dark gray), a pyrex lid (light gray), water (blue), and a piezo transducer (black). (b) The corresponding two-dimensional computational domain $\Omega$ (blue) surrounded by rigid walls $\partial \Omega$ (black) implemented in our numerical model.
Table 1 Model parameters. The parameters are given at temperature

\begin{tabular}{|c|c|c|c|}
\hline \multicolumn{4}{|l|}{ Polystyrene } \\
\hline $\begin{array}{l}\text { Density }{ }^{30} \\
\text { Speed of sound }{ }^{31} \text { (at } 20{ }^{\circ} \mathrm{C} \text { ) } \\
\text { Poisson's ratio }{ }^{32} \\
\text { Compressibility }^{a}\end{array}$ & $\begin{array}{l}\rho_{\mathrm{ps}} \\
c_{\mathrm{ps}} \\
\sigma_{\mathrm{ps}} \\
\kappa_{\mathrm{ps}}\end{array}$ & $\begin{array}{l}1050 \\
2350 \\
0.35 \\
249\end{array}$ & $\begin{array}{l}\mathrm{kg} \mathrm{m}^{-3} \\
\mathrm{~m} \mathrm{~s}^{-1} \\
\mathrm{TPa}^{-1}\end{array}$ \\
\hline \multicolumn{4}{|l|}{ Water } \\
\hline $\begin{array}{l}\text { Density } \\
\text { Speed of sound } \\
\text { Compressibility }^{b} \\
\text { Viscosity } \\
\text { Visc. boundary layer, } 1.97 \mathrm{MHz} \\
\text { Thermal conductivity } \\
\text { Specific heat capacity } \\
\text { Specific heat capacity ratio }{ }^{c} \\
\text { Thermal diffusivity } \\
\text { Thermal expansion coeff. }^{e}\end{array}$ & $\begin{array}{l}\rho_{0} \\
c_{0} \\
\kappa_{0} \\
\eta \\
\delta \\
k_{\text {th }} \\
C_{p} \\
\gamma \\
D_{\text {th }} \\
\alpha\end{array}$ & $\begin{array}{l}998 \\
1495 \\
448 \\
0.893 \\
0.38 \\
0.603 \\
4183 \\
1.014 \\
1.43 \times 10^{-7} \\
2.97 \times 10^{-4}\end{array}$ & $\begin{array}{l}\mathrm{kg} \mathrm{m}^{-3} \\
\mathrm{~m} \mathrm{~s}^{-1} \\
\mathrm{TPa}^{-1} \\
\mathrm{mPa} \mathrm{s} \\
\mu \mathrm{m} \\
\mathrm{W} \mathrm{m}^{-1} \mathrm{~K}^{-1} \\
\mathrm{Jkg}^{-1} \mathrm{~K}^{-1} \\
\mathrm{~m}^{2} \mathrm{~s}^{-1} \\
\mathrm{~K}^{-1}\end{array}$ \\
\hline \multicolumn{4}{|l|}{$50 \%$ glycerol-in-water mixture } \\
\hline $\begin{array}{l}\text { Density } \\
\text { Speed of sound }^{34} \\
\text { Compressibility }^{b} \\
\text { Viscosity } \\
\text { Visc. boundary layer, } 2.27 \mathrm{MHz} \\
\left.\text { Thermal cond. }{ }^{36} \text { (at } 20{ }^{\circ} \mathrm{C}\right) \\
\left.\text { Specific heat cap. }{ }^{37} \text { (at } 1.7^{\circ} \mathrm{C}\right) \\
\text { Specific heat capacity ratio }{ }^{c} \\
\text { Thermal diffusivity } \\
\text { Thermal expansion coeff. }\end{array}$ & $\begin{array}{l}\rho_{0} \\
c_{0} \\
\kappa_{0} \\
\eta \\
\delta \\
k_{\text {th }} \\
C_{p} \\
\gamma \\
D_{\text {th }} \\
\alpha\end{array}$ & $\begin{array}{l}1129 \\
1725 \\
298 \\
5.00 \\
0.79 \\
0.416 \\
3360 \\
1.043 \\
1.10 \times 10^{-7} \\
4.03 \times 10^{-4}\end{array}$ & $\begin{array}{l}\mathrm{kg} \mathrm{m}^{-3} \\
\mathrm{~m} \mathrm{~s}^{-1} \\
\mathrm{TPa}^{-1} \\
\mathrm{mPa} \mathrm{s} \\
\mu \mathrm{m} \mathrm{m} \\
\mathrm{W} \mathrm{m}^{-1} \mathrm{~K}^{-1} \\
\mathrm{~J} \mathrm{~kg}^{-1} \mathrm{~K}^{-1} \\
\mathrm{~m}^{2} \mathrm{~s}^{-1} \\
\mathrm{~K}^{-1}\end{array}$ \\
\hline $\begin{array}{l}{ }^{a} \text { Calculated as } \kappa_{\mathrm{ps}}=\frac{3\left(1-\sigma_{\mathrm{ps}}\right)}{1+\sigma_{\mathrm{ps}}} \frac{(\rho}{(\rho} \\
{ }^{b} \text { Calculated as } \kappa_{0}=1 /\left(\rho_{0} \mathrm{c}_{0}{ }^{2}\right) \text { from } \\
{ }^{c} \text { Calculated from } T_{0} \alpha^{2} /\left(\rho_{0} C_{p} \kappa\right)=\gamma \\
{ }^{d} \text { Calculated as } D_{\mathrm{th}}=k_{\mathrm{th}} /\left(\rho_{0} C_{p}\right) . \\
{ }^{e} \text { Calculated from eqn }(2 \mathrm{c}) .\end{array}$ & ps) & ref. 33. & \\
\hline
\end{tabular}
$T=25^{\circ}$ and taken from the COMSOL Material Library unless explicit stated otherwise.

tracing and velocimetry. ${ }^{7,8}$ This module provides a Lagrangian description of the motion of the particles, each of which is treated as a point particle governed by Newton's law of motion, and thus involving one ordinary differential equation (ODE) for each spatial direction. Consequently, in total $2 N$ ODEs are solved for the particle suspension. The input are the particle masses $m_{j}$ and all forces $\mathrm{F}_{i}\left(\boldsymbol{r}_{j}\right)$ acting on each particle at position $\boldsymbol{r}_{j}$. The ODE for the $j$ th particle with velocity $\boldsymbol{v}_{j}=\mathrm{d} \boldsymbol{r}_{j} / \mathrm{d} t$ is

$$
m_{j} \frac{\mathrm{d} \boldsymbol{v}_{j}}{\mathrm{~d} t}=\sum_{i} \boldsymbol{F}_{i}\left(\boldsymbol{r}_{j}\right)
$$

Neglecting gravitational effects, the forces acting on a particle in our model are the radiation force $\boldsymbol{F}^{\mathrm{rad}}$, eqn (11), and the Stokes drag force $\boldsymbol{F}^{\text {drag }}$, eqn (14). These forces are calculated numerically as described in the following sub-section.

\section{Numerical procedure}

We have used the following sequential procedure to solve the problem numerically in COMSOL:

(i) The first-order acoustic fields of eqn (5), subject to the boundary conditions of eqn (4), are calculated using the predefined Thermoacoustic Physics Interface. 
(ii) The time-averaged second-order flow $\left\langle\boldsymbol{v}_{2}\right\rangle$ is calculated by implementing eqn (9) in the Laminar Flow Physics Interface, modified to include the addition of the time-averaged first-order products from step $(i)$ on the right-hand sides: the right-hand side of eqn ( $9 \mathrm{a})$ is included as a mass source term by adding a so-called weak contribution to the governing equations, $-\frac{1}{2} \int_{\Omega}\left[\partial_{x} \operatorname{Re}\left(\rho_{1} v_{1 x}^{*}\right)+\partial_{y} \operatorname{Re}\left(\rho_{1} v_{1 y}^{*}\right)\right] \tilde{p}_{2} \mathrm{~d} V\left(\tilde{p}_{2}\right.$ being the pressure test function), while the right-hand side of eqn (9b) is added straightforwardly as a body force term. Furthermore, the fourthorder non-linear term $\rho_{0}\left(\left\langle\boldsymbol{v}_{2}\right\rangle \cdot \nabla\right)\left\langle\boldsymbol{v}_{2}\right\rangle$ is kept in the laminar flow equations in COMSOL to enhance numerical stability.

(iii) The acoustic radiation forces are determined using eqn (11) with the first-order fields of step $(i)$.

(iv) Finally, the time-dependent motion of the particles is determined using the COMSOL Particle Tracing Module only taking into account the radiation force of step (iii) and the drag force of eqn (14).

The solution strategy was carried out on a computational mesh large enough for all dependent variables to reach convergence, while taking special care to properly resolve the acoustic boundary layer with an adequate computational mesh, see Section III E. This fine mesh was used when determining the first-order fields and the time-averaged second-order fields. In the subsequent simulation of the time-dependent particle motion, the flow field and radiation forces were interpolated to a coarser mesh to speed up the transient solving procedure substantially.

\section{Computer hardware requirements}

The computation was performed on a DELL Precision 7500 workstation running Windows 7 (64-bit) equipped with 48 GB RAM (1333 MHz) and two hexa-core Intel Xeon X5650 processors of clock frequency $2.66 \mathrm{GHz}$ and $12 \mathrm{MB}$ cache.

When calculating the first-order acoustic fields in step (i), we used the mesh found by the mesh-convergence analysis described in the following subsection, and this resulted in about $3 \times 10^{6}$ degrees of freedom, a calculation time of $4.5 \mathrm{~min}$, and a peak RAM usage of $64 \%$ or $31 \mathrm{~GB}$. The calculation of the secondorder acoustic fields in step (ii) required around $5 \times 10^{5}$ degrees of freedom and took $2 \mathrm{~min}$, while having a peak RAM usage of $19 \%$ or 9 GB. The computation time for steps (iii) and (iv) was less than $15 \mathrm{~s}$ for calculation of 144 particle trajectories of 100 time steps and solved on a coarser mesh resulting in about $9 \times 10^{4}$ degrees of freedom.

\section{E Mesh convergence}

The computational mesh is generated from a maximum element size length $d_{\text {mesh }}$ at the domain boundaries $\partial \Omega$ and a maximum element size in the bulk of the domain $\Omega$ given by $10 d_{\text {mesh }}$. For illustrative purposes, the computational mesh shown in Fig. 3(a) is a coarse mesh with 1204 elements and $d_{\text {mesh }}=20 \delta$, or $\delta / d_{\text {mesh }}=$ 0.05 , where $\delta$ is the boundary layer thickness defined in eqn (7). In order to verify the correctness of the solution, a meshconvergence analysis is required. The solutions are compared for decreasing mesh element size $d_{\text {mesh }}$ to determine the point at which the solution becomes independent of any further decrease of $d_{\text {mesh }}$. We define a relative convergence parameter $C(g)$ for a solution $g$ with respect to a reference solution $g_{\text {ref }}$ taken to be the
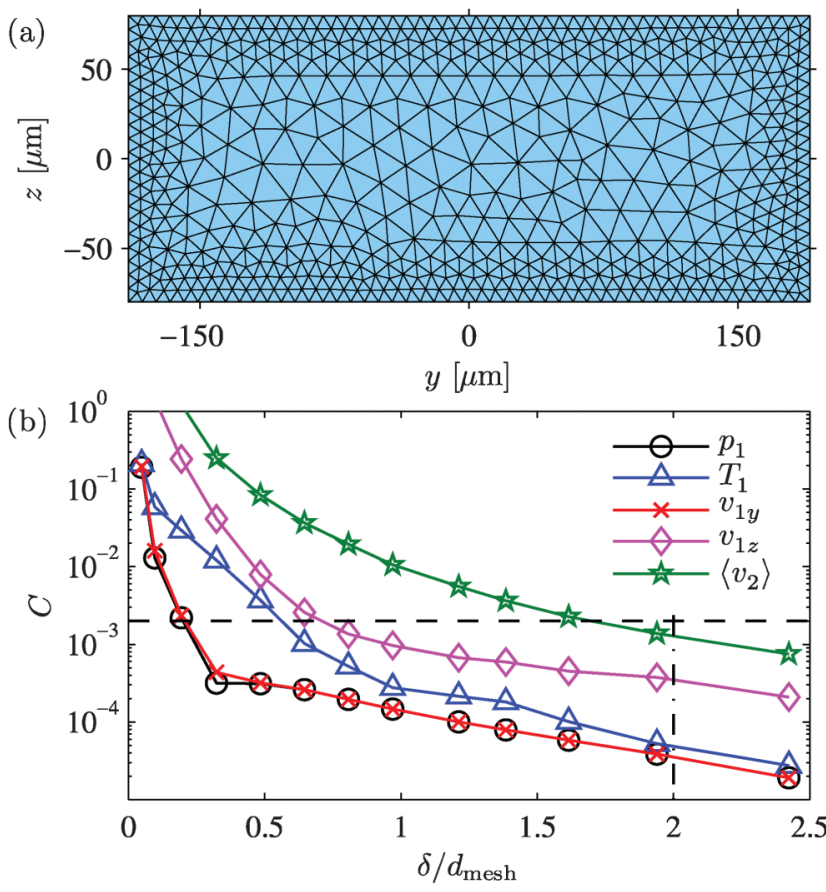

Fig. 3 (a) The computational mesh for a maximum element size of $d_{\text {mesh }}$ $=20 \delta$ at the boundaries, resulting in a coarse mesh with only 1204 triangular elements. (b) Semi-logarithmic plot of the relative convergence parameter $C$, eqn (16), for the physical fields as the size of the mesh elements is decreased. The dashed line indicates a threshold of $C=0.002$, chosen as a trade off between accuracy and computational time. For the second-order velocity field to get below this convergence threshold, a maximum element size of $d_{\text {mesh }}=0.5 \delta$ or $\delta / d_{\text {mesh }}=2.0$ is needed at the boundaries of the domain (dash-dotted line).

solution for the smallest value of $d_{\text {mesh }}$,

$$
C(g)=\sqrt{\frac{\int\left(g-g_{\text {ref }}\right)^{2} \mathrm{~d} y \mathrm{~d} z}{\int\left(g_{\text {ref }}\right)^{2} \mathrm{~d} y \mathrm{~d} z} .}
$$

For $g_{\text {ref }}$ we have chosen $d_{\text {mesh }}=0.3 \delta$ or $\delta / d_{\text {mesh }}=3.3$, which resulted in $2.6 \times 10^{5}$ triangular mesh elements.

The exponential convergence of both first- and second-order fields for $d_{\text {mesh }}<\delta$ shows up as straight lines in the semilogarithmic plots of Fig. 3(b). The time-averaged second-order velocity field $\left\langle\boldsymbol{v}_{2}\right\rangle$ converges considerably slower than the firstorder fields, as it depends on the gradients of the first-order fields, and thus demands better resolution. In order to obtain a relative convergence of the second-order velocity field below 0.002 (dashed line), a maximum element size of $d_{\text {mesh }}=0.5 \delta$ or $\delta / d_{\text {mesh }}=2.0$ is needed at the boundaries. This mesh size, which results in $1.2 \times 10^{5}$ triangular elements, is used for the results presented in this paper.

\section{Results}

The following results are aimed at showing the insensitivity of the horizontal half-wave resonance to the specific form of the ultrasound actuation, at characterizing the first- and secondorder acoustic fields, and at investigating the dependence of the acoustophoretic microparticle motion on system geometry and material parameters. 


\section{A Actuation}

The ultrasound actuation is modeled using the velocity boundary condition in eqn (4c) at the frequency $1.97 \mathrm{MHz}$ to excite the horizontal half-wave resonance in our model system Fig. 2(b). Using the following three actuation modes for the velocity boundary condition $v_{\mathrm{bc}}$ in eqn $(4 \mathrm{c})$,

$$
\begin{aligned}
& v_{\mathrm{bc}}^{A}( \pm w / 2, z)= \pm v_{\mathrm{bc}, 0}^{A}, \\
& v_{\mathrm{bc}}^{B}(y,-h / 2)=v_{\mathrm{bc}, 0}^{B} \sin \left(2 \pi \frac{y}{w}\right), \\
& v_{\mathrm{bc}}^{C}(y,-h / 2)=v_{\mathrm{bc}, 0}^{C}\left(\frac{1}{2}-\frac{y}{w}\right)\left(\frac{1}{2}+\frac{y}{w}\right)^{2},
\end{aligned}
$$

we show the expected result that this resonance is indeed excited regardless of the detailed spatial dependence of $v_{\mathrm{bc}}$ as long as the oscillation frequency equals the resonance frequency.

For all three actuation modes, the amplitude of the velocity boundary conditions is chosen in such a way that the line integral of the absolute value $\left|v_{\mathrm{bc}}\right|$ of the velocity along the perimeter $\partial \Omega$ of the domain $\Omega$ is given in terms of the angular frequency $\omega$ and a characteristic value $d_{0}$ of the displacement of an actuated boundary,
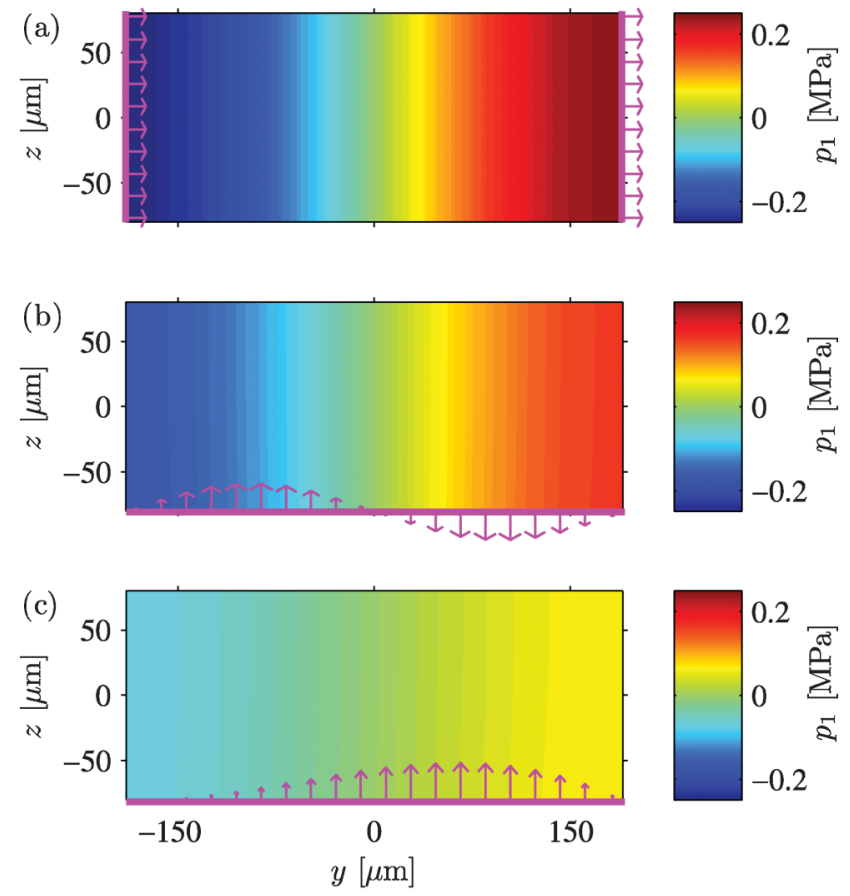

Fig. 4 Three different actuation modes $v_{\mathrm{bc}}$ (magenta arrows) of the water-filled cavity. Color plot of the first-order pressure field $p_{1}$ resulting from the actuation, eqn (17). In all cases the actuation frequency is $1.97 \mathrm{MHz}$, corresponding to the lowest resonance frequency of the cavity, and it is seen that all three actuation modes excite the horizontal half-wave resonance. The pressure amplitude of the resonance mode is (a) $0.24 \mathrm{MPa}$ with side-wall actuation, (b) $0.16 \mathrm{MPa}$ with anti-symmetric bottom-wall actuation, and (c) $0.06 \mathrm{MPa}$ with non-symmetric bottom-wall actuation.

$$
\oint_{\partial \Omega}\left|v_{\mathrm{bc}}^{X}\right| \mathrm{d} \ell=2 h \omega d_{0}, \quad X=A, B, C .
$$

In particular, the normalization constant $2 h$ is chosen so that $v_{\mathrm{bc}, 0}^{A}=\omega d_{0}$, with $d_{0}=0.1 \mathrm{~nm}$, a typical value of displacements, ${ }^{38}$ which results in resonance amplitudes in the range of those measured in typical experiments. ${ }^{7,39-41}$

The first-order pressure fields resulting from the three different actuation modes are shown in Fig. 4. It is seen that all of the actuation modes excite the horizontal half-wave $1.97-\mathrm{MHz}$ resonance. Although the velocity boundary conditions have been normalized, the amplitude of the resonance is different for each of the three actuation modes, i.e. each actuation mode couples to the resonance mode with its own strength. In the studies presented in the rest of this paper, we have used the velocity boundary condition eqn (17a), shown in Fig. 4(a), due to its simplicity and strong coupling to the resonance mode.

\section{B First-order fields}

We now turn to a study of the first-order fields resulting from the velocity boundary condition eqn (17a) and Fig. 4(a). In Fig. 5, color plots of the pressure $p_{1}$, temperature $T_{1}$, horizontal velocity

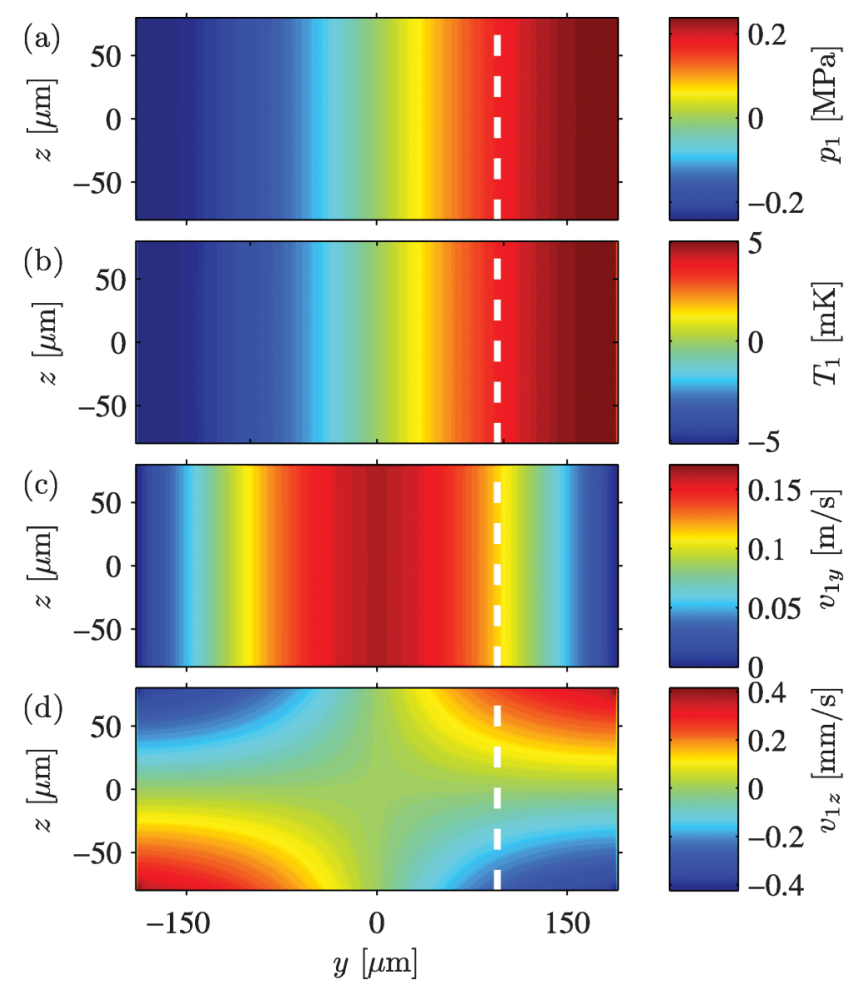

Fig. 5 Color plots of the amplitudes of the oscillating first-order fields in the water-filled channel at the horizontal standing half-wave $1.97-\mathrm{MHz}$ resonance excited by velocity boundary condition eqn (17a): (a) pressure $p_{1}$, identical to panel (a) in Fig. 4, (b) temperature $T_{1}$, (c) horizontal velocity $v_{1 y}$, and (d) vertical velocity $v_{1 z}$. The horizontal velocity is much larger than the vertical velocity, arising because of the interaction of the acoustic resonance with the bottom and top walls. The sub-micrometer thin viscous boundary cannot be seen on the $100-\mu \mathrm{m}$ scale of the plot. The dashed white lines indicate the domain for the line plots in Fig. 6. 

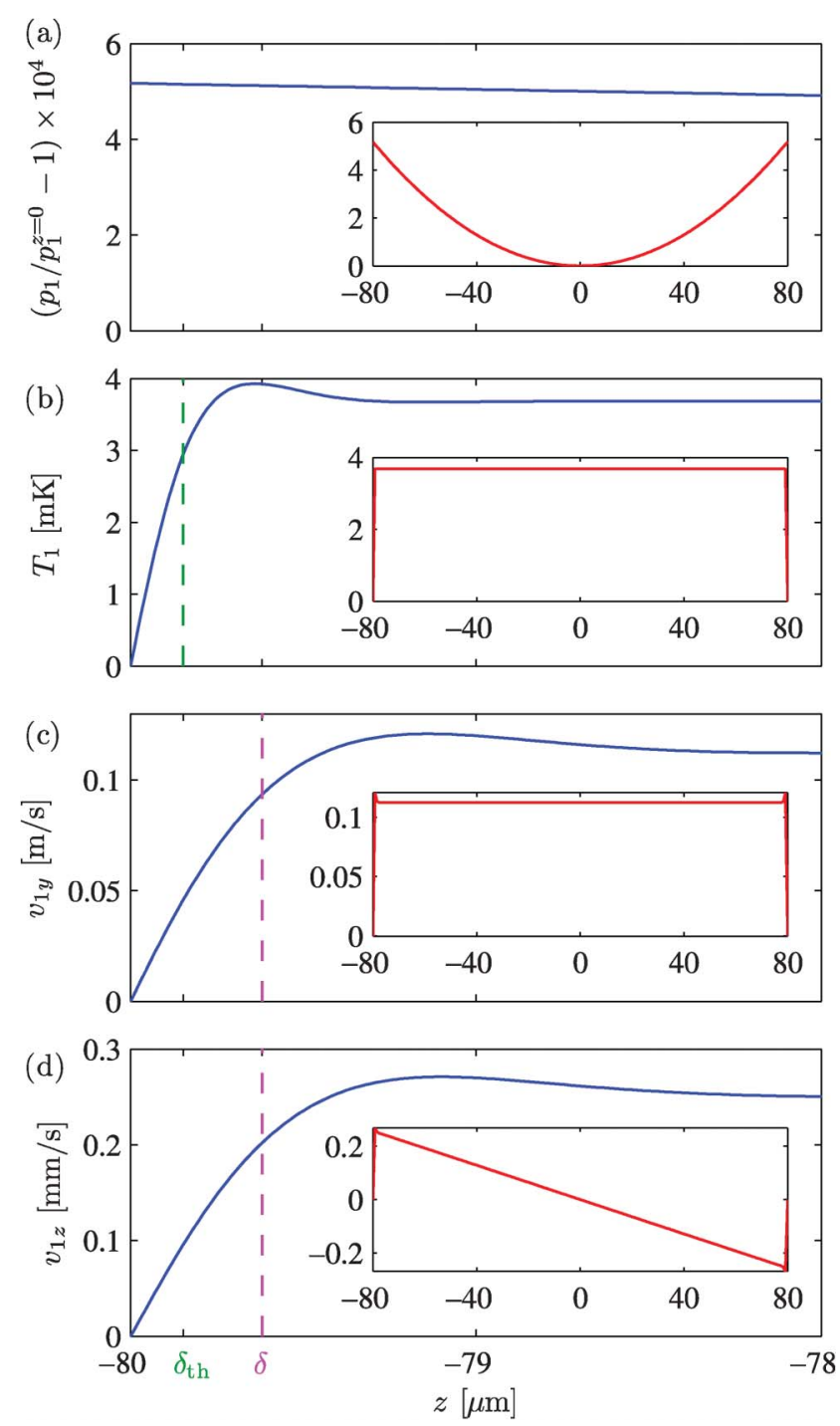

Fig. 6 Line plots, along the dashed white lines at $y=w / 4$ shown in Fig. 5, of the amplitudes of the oscillating first-order fields: (a) relative pressure change $p_{1} / p_{1}^{z=0}-1$, (b) temperature $T_{1}$, (c) horizontal velocity $v_{1 y}$, and (d) vertical velocity $v_{1 z}$. The main plots (blue curves) show the field amplitudes close to the bottom wall, while the insets (red curves) show the field amplitudes along the entire height of the channel. The characteristic length scales of the thermal and viscous boundary layers, $\delta_{\text {th }}$ and $\delta$, are indicated by the green and magenta dashed lines, respectively. $T_{1}, v_{1 y}$, and $v_{1 z}$ all show marked variations on the length scale of the boundary layer, while $p_{1}$ and $v_{1 z}$ only show variations across the full height of the domain.

$v_{1 y}$, and vertical velocity $v_{1 z}$ are shown. The amplitudes and structures of $p_{1}, T_{1}$, and $v_{1 y}$ relate to the acoustic resonance, while $v_{1 z}$ arises due to the viscous interaction of the horizontal half-wave resonance in the bulk with the bottom and top walls. Consequently, the magnitude of $v_{1 z}$ is insignificant compared to the magnitude of $v_{1 y}$. The amplitudes of $p_{1}$ and $T_{1}$ have the same spatial structure, shifted horizontally by $\lambda / 4$ with respect to the spatial structure of $v_{1 y}$.

In Fig. 6 the amplitudes of the first-order fields are plotted along the dashed white lines at $y=w / 4$ shown in Fig. 5. In Fig. 6(a) we have plotted the relative pressure change, $p_{1} / p_{1}^{z=0}-1$, with respect to the pressure amplitude at the center $(y, z)=(w / 4,0)$. This relative change is in the order of $10^{-4}$, implying that $p_{1}$ is nearly independent of $z$. In particular, $p_{1}$ shows no marked variation on the length scale of the boundary layer thickness $\delta$ as opposed to the velocity $v_{1 y}$ and temperature $T_{1}$ of Fig. 6 (b)-(c). To fulfill the boundary conditions in eqn (4), the latter two decrease from their bulk values to zero at the wall over a few times $\delta_{\text {th }}$ and $\delta$, respectively, which defines the thicknesses of the thermal and viscous boundary layers, respectively (dashed lines in Fig. 6). Further, also $v_{1 z}$ increases from zero at the wall, but then it exhibits a slow linear decrease outside the boundary layer, Fig. 6(d). $T_{1}, v_{1 y}$, and $v_{1 z}$ all overshoot slightly before settling at their respective bulk values, an effect similar to that observed in the classical problem of a planar wall executing in-plane oscillations. ${ }^{20}$ While $T_{1}$ and $v_{1 y}$ show no variations in the height of the channel outside the boundary layers, $p_{1}$ and $v_{1 z}$ do so, with $p_{1}$ being symmetric (nearly parabolic) and $v_{1 z}$ being antisymmetric (nearly linear). These variations of $p_{1}$ and $v_{1 z}$ result from the viscous interaction between the horizontal acoustic resonance and the bottom and top wall, bounding the acoustic resonance fields.

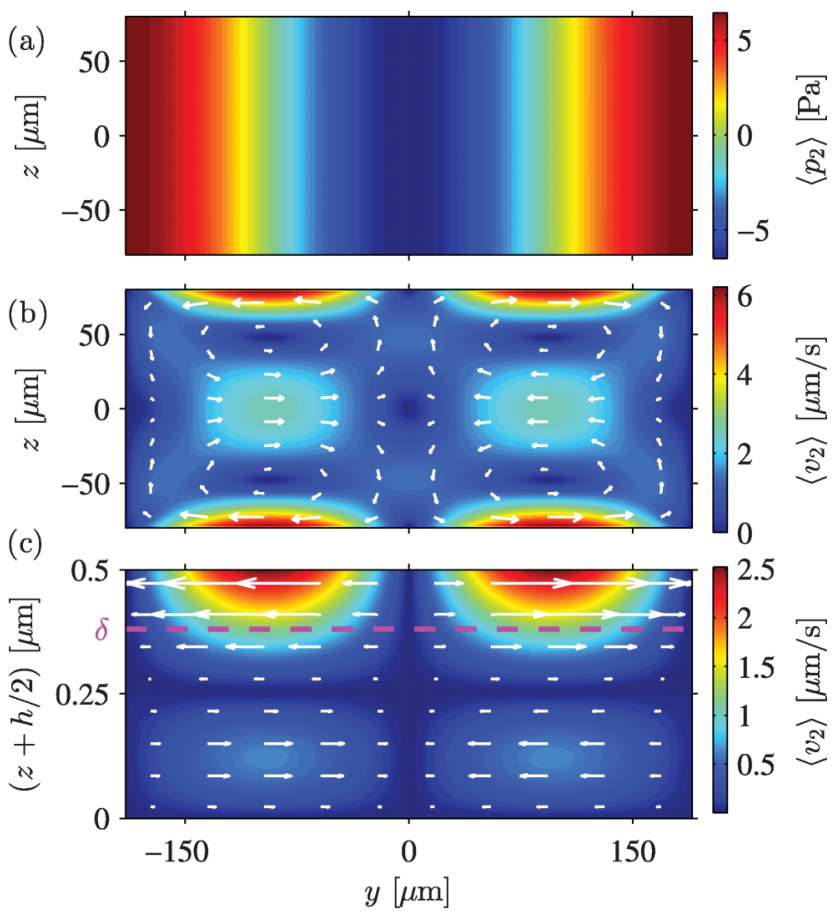

Fig. 7 Time-averaged second-order fields in the water-filled channel excited in the horizontal half-wave 1.97-MHz resonance by the side-wall actuation shown in Fig. 4(a) and driven by the first-order fields plotted in Fig. 5. (a) Color plot of the time-averaged second-order pressure $\left\langle p_{2}\right\rangle$ with a magnitude approximately $2.5 \times 10^{-5}$ times smaller than the amplitude of the oscillating first-order pressure $p_{1}$ in Fig. 5(a). (b) Vector plot (white arrows) of the time-averaged second-order streaming velocity $\left\langle\boldsymbol{v}_{2}\right\rangle$ and color plot of its magnitude $\left\langle v_{2}\right\rangle$. Four bulk (Rayleigh) streaming rolls are clearly seen having the maximum speed near the top and bottom walls. (c) Zoom-in on the $0.4-\mu$ m-thick boundary layer near the bottom wall exhibiting the two boundary (Schlichting) streaming rolls that drive the bulk (Rayleigh) streaming rolls. 


\section{Second-order fields and acoustic streaming}

As described by eqn (9) the non-linear interactions of the firstorder fields give rise to time-averaged second-order fields, resulting in a static pressure field and a steady velocity field as shown in Fig. 7 . The time-averaged second-order fields exhibit a spatial oscillation in the horizontal $y$-direction of wave length $\lambda / 2$. This is reminiscent of the spatial period doubling for the classical infinite parallel-plate system of Fig. 1 .

The magnitude of the time-averaged second-order pressure $\left\langle p_{2}\right\rangle$ in Fig. 7(a) is approximately $2.5 \times 10^{-5}$ times smaller than the amplitude of the oscillating first-order pressure in Fig. 5(a). The time-averaged velocity field $\left\langle\boldsymbol{v}_{2}\right\rangle$ in Fig. 7(b) contains four bulk (Rayleigh) streaming rolls. The streaming velocity is maximum close to the top and bottom wall and has two local maxima on the horizontal center axis $z=0$, where opposite pairs of bulk rolls meet. The solution in Fig. 7(b) is similar to Rayleigh's analytical solution ${ }^{17,20}$ sketched in Fig. 1, but it deviates on the following two points: (i) as we consider a rectangular geometry and not parallel plates, the velocity is forced to be zero at the side walls, which slightly slows down the rotational flow of the streaming rolls, and (ii) as we are not in the limit $h \ll \lambda$, the strength of the streaming rolls decreases slightly before meeting in the center of the channel, which results in a lower magnitude of the streaming velocity in the horizontal center plane than predicted by Rayleigh.

Fig. 7(c) shows a zoom-in on the $0.4-\mu \mathrm{m}$-thick viscous boundary layer close to the bottom wall containing two boundary (Schlicting) streaming rolls. These boundary rolls are very elongated in the horizontal direction; the $z$-axis in Fig. 7(c) is stretched nearly a factor $10^{3}$ relative to the $y$-axis. It is important to mention that the boundary streaming rolls are generated by the non-linear interactions of the first-order fields inside the boundary layer, whereas the bulk streaming rolls are driven by the boundary streaming rolls and not by the non-linear interaction of the first-order fields in the bulk. The time-averaged second-order velocity $\left\langle\boldsymbol{v}_{2}\right\rangle$ is zero at the bottom wall, thus fulfilling the boundary conditions eqn (4b), while the maximum of its horizontal component $v_{2 y}{ }^{\text {bnd }}=6.42 \mu \mathrm{m} \mathrm{s}^{-1}$ is reached at a distance of approximately $3 \delta$ from the wall. The maximum bulk amplitude $U_{1}=0.162 \mathrm{~m} \mathrm{~s}^{-1}$ of the horizontal first-order velocity component, $v_{1 y}$ shown in Fig. 5(c), is reached at the channel center $y=0$. From this we calculate the characteristic velocity ratio $\Psi=c_{0} v_{2 y}^{\text {bnd }} / U_{1}^{2}=0.367$, which deviates less than $3 \%$ from the value $\Psi=3 / 8=0.375$ of the parallel-plate model eqn (10).

\section{Particle tracing simulations}

In most experimental microfluidic flow visualization techniques, tracer particles are employed. ${ }^{42}$ To mimic this and to ease comparison with experiment, we have performed particle tracing simulations using the technique described in Section III B. In all simulations, we have studied the motion of 144 polystyrene microparticles suspended in water and distributed evenly at the initial time $t=0$ as shown in Fig. 8(a).

In Fig. 8(b)-(f), the particle trajectories after $10 \mathrm{~s}$ of acoustophoretic motion of the 144 microparticles are shown. Within each panel, all particles have the same diameter $2 a$, but the particle size is progressively enlarged from one panel to the next: (b) $2 a=0.5 \mu \mathrm{m}$, (c) $1 \mu \mathrm{m}$, (d) $2 \mu \mathrm{m}$, (e) $3 \mu \mathrm{m}$, and (f) $5 \mu \mathrm{m}$.
For the smallest particles, panels (b) and (c), the drag force from the acoustic streaming dominates the particle motion, and the characteristic streaming flow rolls are clearly visualized. For the larger particles, panels (e) and (f), the acoustic radiation force dominates the particle motion, and the particle velocity $\boldsymbol{u}$ is nearly horizontal with the sinusoidal spatial dependence given by $u_{y}(y)=$ $F_{1 \mathrm{D}}^{\mathrm{rad}}(y) /(6 \pi \eta a)$ found from eqn (13a). This results in a focusing motion of the particles towards the vertical pressure nodal plane at $y=0$. Panels (d) and (e) show an intermediate regime where drag and radiation forces are of the same order of magnitude.

At the nodal plane $y=0$ the radiation forces are zero, and consequently for times $t$ larger than $10 \mathrm{~s}$ all particles in panel (f) that have reached $y=0$ end up at $(y, z)=(0, \pm h / 2)$ due to the weak but non-zero streaming-induced drag forces.

The cross-over from one acoustophoretic behavior to the other as a function of particle size, with a critical particle diameter of $2 \mu \mathrm{m}$ found in Fig. 8(d), is in agreement with the following scaling argument: ${ }^{16}$ If we assume a force balance between the radiation force and the drag force from acoustic streaming, $\boldsymbol{F}^{\mathrm{rad}}=-\boldsymbol{F}^{\mathrm{drag}}$, keeping a given particle fixed $(\boldsymbol{u}=\mathbf{0})$, and if we estimate the magnitude of the streaming velocity to be given by eqn (10) as $\left\langle v_{2}\right\rangle=\Psi U_{1}^{2} / c_{0}$, where $\Psi$ is a geometrydependent factor of order unity, then eqn (13) and (14) lead to

$$
\pi a_{c}^{3} q \rho_{0} U_{1}^{2} \Phi \approx 6 \pi \eta a_{c} \Psi \frac{U_{1}^{2}}{c_{0}},
$$

where $a_{c}$ is the critical particle radius. Thus, as found in eqn (16), the critical particle diameter $2 a_{c}$ becomes

$$
2 a_{c}=\sqrt{12 \frac{\Psi}{\Phi}} \delta \approx 2.0 \mu \mathrm{m} .
$$

The value is calculated using $\Psi=0.375$, valid for a planar wall (eqn (10)), and $\Phi=0.165$, obtained for polystyrene particles with diameters between $0.5 \mu \mathrm{m}$ and $5 \mu \mathrm{m}$ in water obtained from eqn (13b) using the parameter values from Table 1 . The relation eqn (20) for the critical cross-over particle diameter is important for designing experiments relying on specific acoustophoretic behaviors as function of particle size. Channel geometry enters through the factor $\Psi$, particle and liquid material parameters through $\Phi$, and liquid parameters and frequency through the boundary layer thickness $\delta$.

\section{E Streaming for an increased aspect ratio}

As an example of how geometry affects the acoustophoretic motion of polystyrene microparticles, we study here the consequences of increasing the aspect ratio of the channel cross-section from $h / w=0.42$ to 2 keeping all other parameters fixed. As illustrated in Fig. 9(a), the streaming velocity field is only significant close to the top and bottom of the channel for the large aspect ratio $h / w=2$. This happens because given enough vertical space, the vertical extension $\Delta$ of the streaming roll is identical to the horizontal one, which is $\Delta=\lambda / 4$. For the horizontal half-wave resonance in a channel of aspect ratio $h / w=$ 2 we have $\lambda=2 w=h$, which implies $\Delta=h / 4$, and we therefore expect a streaming-free region with a vertical extent of $h-2(h / 4)$ $=h / 2$ around the center of the channel, which indeed is seen in Fig. 9(a). 

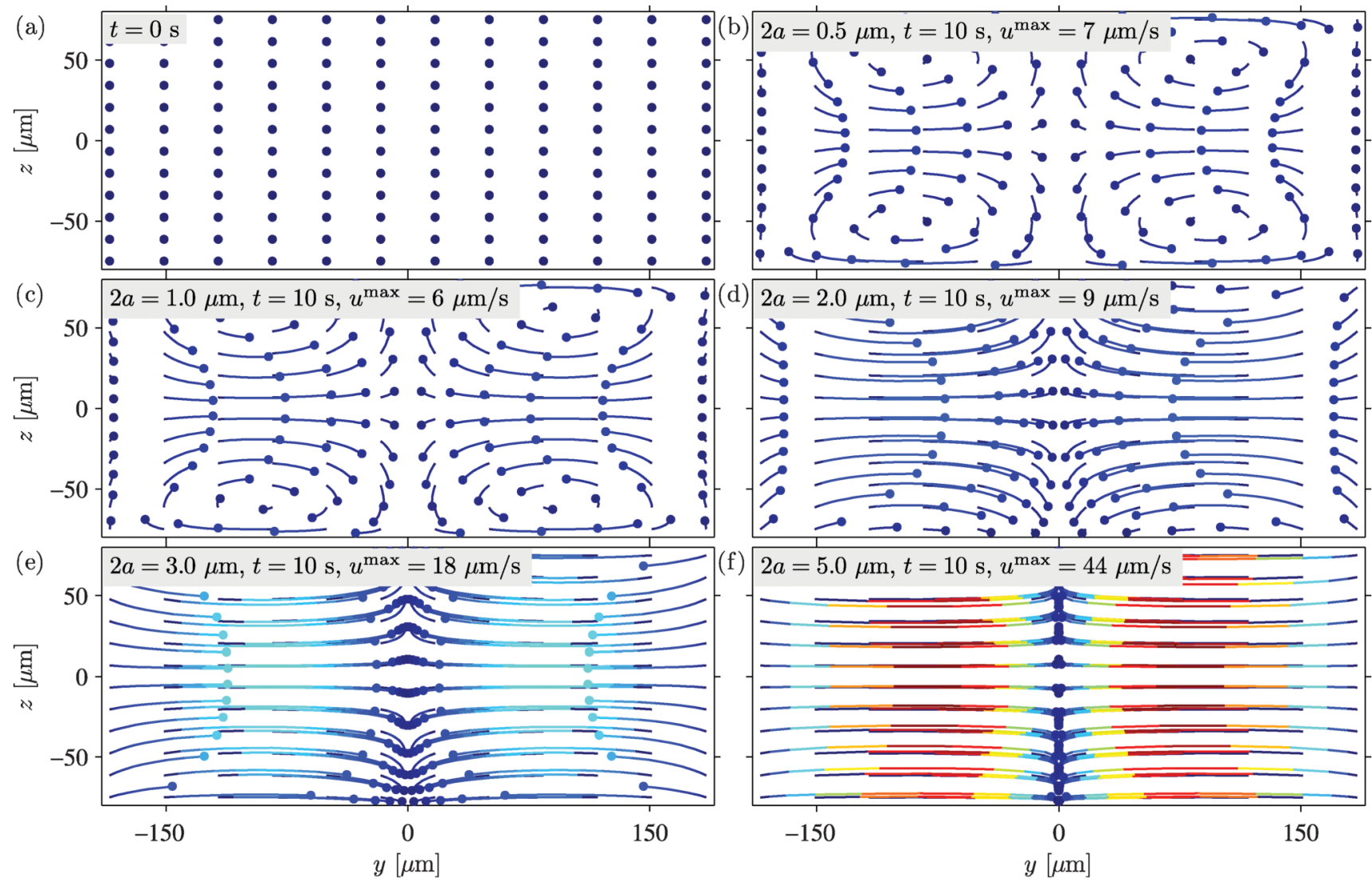

Fig. 8 (a) The starting positions (dots) of 144 evenly distributed particles at $t=0 \mathrm{~s}$ in the computational domain at the onset of the horizontal halfwave 1.97-MHz resonance shown in Fig. 5 and 7. The following five panels show the trajectories (colored lines) and positions (dots) that the particles have reached by acoustophoresis at $t=10 \mathrm{~s}$ for five different particle diameters: (b) $0.5 \mu \mathrm{m}$, (c) $1 \mu \mathrm{m}$, (d) $2 \mu \mathrm{m}$, (e) $3 \mu \mathrm{m}$, and (f) $5 \mu \mathrm{m}$. The colors indicate the instantaneous particle velocity $u$ ranging from $0 \mu \mathrm{m} \mathrm{s}^{-1}$ (dark blue) to $44 \mu \mathrm{m} \mathrm{s}^{-1}$ (dark red). The lengths of the trajectories indicate the distance covered by the particles in $10 \mathrm{~s}$. Streaming-induced drag dominates the motion of the smallest particles, which consequently are being advected along the acoustic streaming rolls of Fig. 7(b). In contrast, the acoustic radiation force dominates the motion of the larger particles, which therefore are forced to the vertical nodal plane at $y=0$ of the first-order pressure $p_{1}$ shown in Fig. 5(a).

As a consequence, the acoustophoretic motion of particles in the center region is controlled by the radiation force. This is illustrated in Fig. 9(b), where trajectories of small 1- $\mu \mathrm{m}$-diameter particles are shown. For $-h / 4<z<h / 4$ their motion is similar to the radiationforce dominated motion of the larger 5 - $\mu$ m-diameter particles moving in the shallow channel with $h / w=0.42$ as shown in Fig. 8(f). Near the top and bottom walls, the $1 \mu \mathrm{m}$ diameter particles exhibit the usual small-particle streaming-induced motion.

Clearly, geometry can be used to obtain more control of the acoustophoretic motion of suspended particles in microchannels.

\section{F Streaming in a high-viscosity buffer}

According to eqn (20), the critical particle diameter for crossover between radiation-dominated and streaming-dominated acoustophoretic motion is proportional to the boundary layer thickness $\delta=\sqrt{2 \eta /\left(\rho_{0} \omega\right)}$. Obviously, viscosity can also be used to control acoustophoresis. We therefore studied the effects of replacing water $(\eta=1 \mathrm{mPa}$ s) with glycerol mixtures, in particular the $50 \%$ glycerol-in-water mixture $(\eta=5 \mathrm{mPa} \mathrm{s})$, for which the relevant material parameters are listed in Table 1.

First, to ensure comparable conditions, we wanted to excite the horizontal half-wave resonance in the glycerol-in-water system. As the speed of sound of the glycerol mixture is $15 \%$ larger than that of water, we found the resonance frequency to be $f=c_{0} /(2 w)=2.27 \mathrm{MHz}$. This frequency was used in the velocity boundary condition eqn (17a) to calculate the results shown in Fig. 10 for the first-order pressure field, the time-averaged second-order streaming velocity field, and particle trajectories for 5 - $\mu \mathrm{m}$-diameter polystyrene particles.

The glycerol-in-water and the water system are actuated with the same boundary velocity given in eqn (18), but the difference in viscosity of the two liquids leads to different acoustic responses. The amplitude of the induced first-order resonance pressure is reduced by a factor of 2.6 from $0.243 \mathrm{MPa}$ in the lowviscosity water of Fig. 5(a) to $0.094 \mathrm{MPa}$ in the high-viscosity glycerol mixture of Fig. 10(a). Likewise, the induced streaming velocity $\left\langle v_{2 y}^{\text {bnd }}\right\rangle$ near the boundary is reduced by a factor of 15 from $6.42 \mu \mathrm{m} \mathrm{s}^{-1}$ in water, Fig. 7(b), to $0.43 \mu \mathrm{m} \mathrm{s}^{-1}$ in glycerolin-water, Fig. 10(b). In contrast, given the validity of Rayleigh's streaming theory, the velocity ratio $\Psi=c_{0}\left\langle v_{2 y}^{\text {bnd }}\right\rangle / U_{1}^{2}$ should be independent of viscosity. For the glycerol-in-water mixture it is 0.336 deviating $8 \%$ from the value in water, see Section IV C, and $10 \%$ from the Rayleigh value $3 / 8$ of eqn (10). The significant difference in the numerically determined values of $\Psi$ for water and glycerol-in-water points to the inadequacy of the Rayleigh 

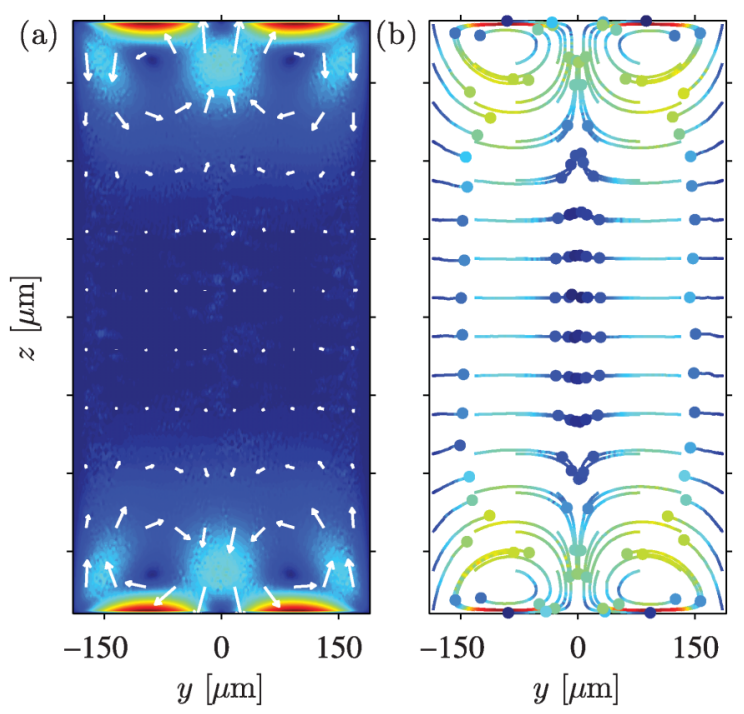

Fig. 9 Acoustophoresis in a high-aspect-ratio channel. The setup is identical to Fig. 4(a) except that for the fixed width $w=0.38 \mathrm{~mm}$ the channel height $h$ has been increased from $0.16 \mathrm{~mm}$ (aspect ratio $h / w=$ 0.42 ) to $0.76 \mathrm{~mm}$ (aspect ratio $h / w=2$ ). (a) Vector plot (white arrows), similar to Fig. 7(b), of the time-averaged second-order streaming velocity $\left\langle\boldsymbol{v}_{2}\right\rangle$ and color plot [from $0 \mu \mathrm{m} \mathrm{s}^{-1}$ (dark blue) to $4.2 \mu \mathrm{m} \mathrm{s}^{-1}$ (dark red)] of its magnitude. (b) Particle tracing plot for $1-\mu \mathrm{m}$-diameter polystyrene particles corresponding to Fig. 8(c) but for time $t=100 \mathrm{~s}$, aspect ratio $h / w=2$, and velocity ranging from $0 \mu \mathrm{m} \mathrm{s}^{-1}$ (dark blue) to $3.3 \mu \mathrm{m} \mathrm{s}^{-1}$ (dark red). In this high aspect-ratio geometry the acoustic streaming flow rolls are located near the top and bottom walls leaving the center region nearly streaming free.

theory in a rectangular channel. This is perhaps not a surprise, as this theory is derived for an infinite parallel-plate channel.

In Fig. $10(\mathrm{c})$ is shown that the viscous boundary-layer thickness in the glycerol-in-water mixture at $2.27 \mathrm{MHz}$ is $\delta=$ $0.79 \mu \mathrm{m}$, a factor 2.1 larger than the value $\delta=0.38 \mu \mathrm{m}$ in water at $1.97 \mathrm{MHz}$ shown in Fig. 7(c). As the two resonance frequencies only differ by $10 \%$, the change in the boundarylayer thickness is mainly due to the viscosity ratio, $\sqrt{5 \mathrm{mPa} \mathrm{s}} / \sqrt{1 \mathrm{mPas}} \approx 2.2$.

Finally, from eqn (10) using $\Psi=3 / 8$ and $\Phi=0.031$, we calculated the critical particle diameter to be $2 a_{c}=9.5 \mu \mathrm{m}$ for the cross-over from radiation-dominated to streaming-dominated acoustophoretic motion in the glycerol-in-water system. This value explains why the particle trajectories for the 5- $\mu \mathrm{m}$-diameter polystyrene particles in Fig. 10(d) appear to be much more influenced by the acoustic streaming rolls, compared to the same-sized particles in water, Fig. 8(f). Instead, Fig. 10(d) resembles more the motion of the $1-\mu \mathrm{m}$-diameter particles in water, Fig. 8(c). This resemblance can be quantified by the ratio $a / a_{c}$ : for 5 - $\mu \mathrm{m}$-diameter particles in the glycerol-in-water mixture it is 0.52 , while for $1-\mu \mathrm{m}$-diameter particles in pure water it is 0.50 , only $4 \%$ lower. Note that because of the reduction in streaming velocity by the above-mentioned factor of 15 , we have chosen to follow the particles in the glycerolin-water mixture for $150 \mathrm{~s}$ and in water only for $10 \mathrm{~s}$.

\section{Concluding discussion}

The finite element method was successfully used to model the acoustophoretic motion of microparticles inside a microchannel
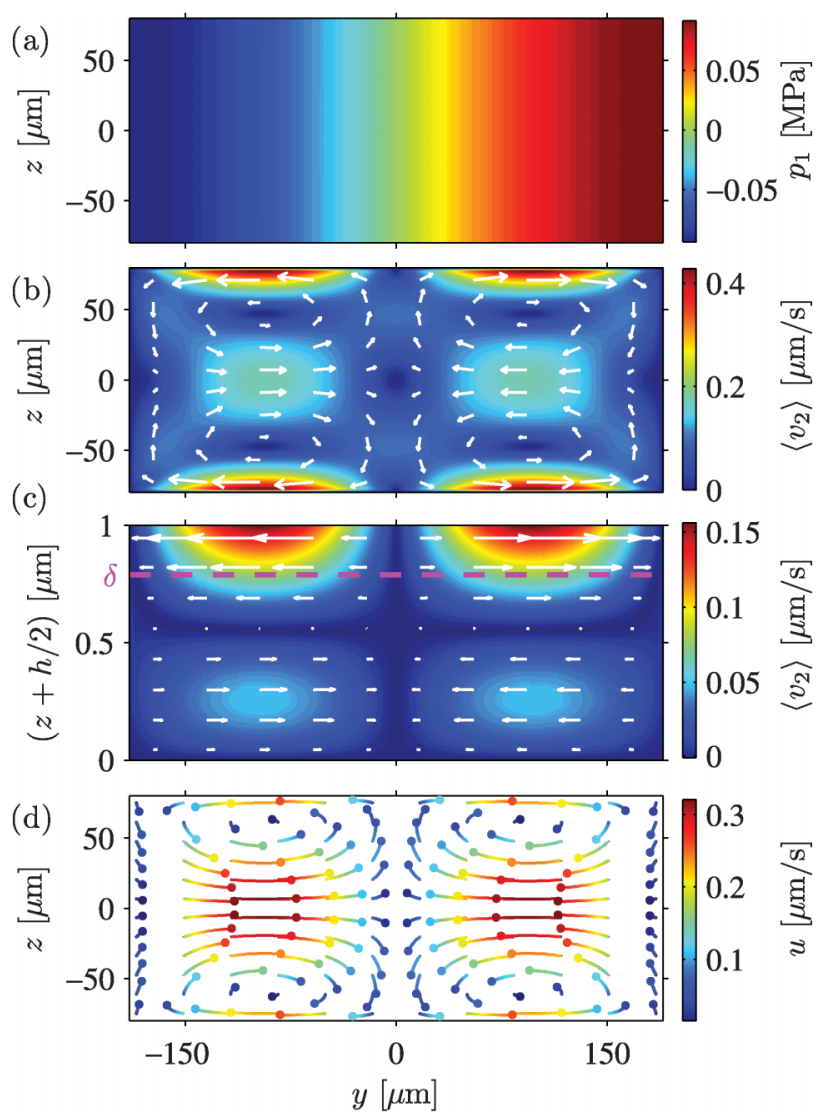

Fig. 10 Acoustophoresis in a 50\% glycerol-in-water mixture. The setup is identical to Fig. 4(a) except that the resonance frequency is increased to $f=c_{0} /(2 w)=2.27 \mathrm{MHz}$. (a) Color plot of the pressure $p_{1}$ showing the horizontal half-wave resonance. (b) Vector plot (white arrows) of the time-averaged second-order streaming velocity $\left\langle\boldsymbol{v}_{2}\right\rangle$ and color plot of its magnitude corresponding to Fig. 7(b). (c) Zoom-in on the $0.4-\mu \mathrm{m}$-thick boundary layer near the bottom wall corresponding to Fig. 7(c). (d) Particle tracing plot for $5-\mu \mathrm{m}$-diameter polystyrene particles corresponding to Fig. 8(f) but for $150 \mathrm{~s}$. Contrary to the water-filled channel in Fig. $8(\mathrm{f})$, the motion of the 5 - $\mu \mathrm{m}$-particles in the more viscous glycerol-inwater mixture are dominated by the streaming-induced drag, whereby the particle trajectories end up looking more like those of the smaller $1-\mu \mathrm{m}$ diameter particles Fig. 8(c).

subject to a transverse horizontal half-wave ultrasound resonance. The motion is due to the combined effect of Stokes drag from the time-averaged second-order streaming flow and the acoustic radiation forces. To achieve this, the first-order acoustic field of a standing wave was determined inside a microchannel cavity by solving the linearized compressional Navier-Stokes equation, the continuity equation, and the heat equation, while resolving the boundary layers near rigid walls. The first-order field was then used to determine the streaming flow and the acoustic radiation forces, and from this the time-dependent trajectories of an ensemble of non-interacting microparticles was calculated.

A main result is the characterization of the cross over from streaming-dominated to radiation-dominated acoustophoretic microparticle motion as a function of particle diameter, geometry, and viscosity. Using a water-filled shallow microchannel as the base example, we demonstrated how to get rid of streaming effects in the center region of a microchannel by 
increasing the height-to-width ratio. In contrast, by replacing water by a $50 \%$ glycerol-in-water mixture, we demonstrated how to enhance the streaming effects. The former study may form a good starting point for designing streaming-free devices for handling of sub-micrometer particles, such as small cells, bacteria, and viruses, and thus supporting concurrent experimental efforts to suppress streaming, e.g., through averaging over alternating actuation frequencies. ${ }^{43}$ The latter study is pointing in the direction of developing devices with improved mixing capabilities by enhancing streaming. ${ }^{44,45}$ We have thus shown that our simulation tool has a great potential for enabling improved design of acoustofluidic devices.

An important next step is to obtain a direct experimental verification of our numerical simulation. As the relative uncertainty of measured acoustophoretic particle velocities in current experimental acoustofluidics is $5 \%$ or better, ${ }^{8}$ it is within reach to obtain such an experimental verification. A problem is of course that the streaming fields calculated in this work are in the vertical plane, which is perpendicular to the usual horizontal viewing plane, and thus specialized 3-dimensional visualization techniques are required such as stereoscopic micro particleimage velocimetry ${ }^{42,46}$ or astigmatism particle tracking velocimetry. ${ }^{47}$ But even if such $3 \mathrm{D}$-methods are complex to carry out, it would be worth the effort given the great use of having a wellverified numerical model of acoustophoretic particle motion.

Given a successful experimental verification, it would clearly be valuable to extend the numerical model. One obvious step, which is not conceptually difficult, but which would require significant computational resources, would be to make a full 3D-model taking the elastic properties of the chip surrounding the microchannel into account. The relevance of such an extension lies in the sensitivity of the acoustic streaming on the boundary conditions. Only a full acousto-elastic theory would supply realistic and accurate boundary conditions. Another class of obvious model extensions deals with the modeling of the particle suspension. A trivial extension would be to include gravity and buoyancy, but more importantly and much more difficult would be the inclusion of particle-particle and particle-wall interactions that are neglected in the present work. These many-particle effects include, e.g., the generation of streaming flow in the boundary layer of each particle ${ }^{48}$ and not just the boundary layer of the wall. After such an extension, our model could be used together with high-precision experiments as a new and better research tool to study and clarify the many yet unsolved problems with particleparticle and particle-wall interactions in acoustofluidics.

The above-mentioned applications all demonstrate that our numerical model is both timely and has a huge potential within device design and studies of basic physical aspects of acoustophoresis.

\section{Acknowledgements}

This research was supported by the Danish Council for Independent Research, Technology and Production Sciences, Grants No. 274-09-0342 and No. 11-107021.

\section{References}

1 J. Friend and L. Y. Yeo, Rev. Mod. Phys, , 2011, 83, 647-704.

2 H. Bruus, J. Dual, J. Hawkes, M. Hill, T. Laurell, J. Nilsson, S. Radel, S. Sadhal and M. Wiklund, Lab Chip, 2011, 11, 3579-3580.
3 A. Lenshof, C. Magnusson and T. Laurell, Lab Chip, 2012, 12, $1210-1223$.

4 M. Gedge and M. Hill, Lab Chip, 2012, 12, 2998-3007.

5 H. Bruus, Lab Chip, 2012, 12, 1014-1021.

6 M. Wiklund, R. Green and M. Ohlin, Lab Chip, 2012, 12, 2438-2451.

7 R. Barnkob, P. Augustsson, T. Laurell and H. Bruus, Lab Chip, 2010, 10, 563-570.

8 P. Augustsson, R. Barnkob, S. T. Wereley, H. Bruus and T. Laurell, Lab Chip, 2011, 11, 4152-4164.

9 K. Yosioka and Y. Kawasima, Acustica, 1955, 5, 167-173.

10 L. P. Gorkov, Soviet Physics - Doklady, 1962, 6, 773-775.

11 M. Settnes and H. Bruus, Phys. Rev. E, 2012, 85, 016327.

12 K. Frampton, S. Martin and K. Minor, Appl. Acoust., 2003, 64, 681-692.

13 M. Hamilton, Y. Ilinskii and E. Zabolotskaya, J. Acoust. Soc. Am., 2003, 113, 153-160.

14 J. F. Spengler, W. T. Coakley and K. T. Christensen, AIChE J., 2003, 49, 2773-2782.

15 S. M. Hagsäter, T. G. Jensen, H. Bruus and J. P. Kutter, Lab Chip, 2007, 7, 1336-1344.

16 H. Bruus, Lab Chip, 2012, 12, 1578-1586.

17 L. Rayleigh, Philos. Trans. R. Soc. London, 1884, 175, 1-21.

18 H. Schlichting, Physik Z, 1932, 33, 327-335.

19 J. Lighthill, J. Sound Vib., 1978, 61, 391-418.

20 L. D. Landau and E. M. Lifshitz, Fluid Mechanics, Pergamon Press, Oxford, 2nd edn, 1993, vol. 6, Course of Theoretical Physics.

21 H. Lei, D. Henry and H. BenHadid, Appl. Acoust., 2011, 72, 754-759.

22 M. K. Aktas and B. Farouk, J. Acoust. Soc. Am., 2004, 116, 2822-2831

23 P. M. Morse and K. U. Ingard, Theoretical Acoustics, Princeton University Press, Princeton NJ, 1986.

24 A. D. Pierce, Acoustics, Acoustical Society of America, Woodbury, 1991.

25 D. T. Blackstock, Physical acoustics, John Wiley and Sons, Hoboken NJ, 2000.

26 L. D. Landau and E. M. Lifshitz, Statistical physics, Part 1, Butterworth-Heinemann, Oxford, 3rd edn, 1980, vol. 5.

27 W. L. Nyborg, J. Acoust. Soc. Am., 1953, 25, 68-75.

28 M. Koklu, A. C. Sabuncu and A. Beskok, J. Colloid Interface Sci., 2010, 351, 407-414.

29 COMSOL Multiphysics 4.2a, www.comsol.com, 2012.

30 CRCnetBASE Product, CRC Handbook of Chemistry and Physics, Taylor and Francis Group, www.hbcpnetbase.com/, 92nd edn, 2012.

31 L. Bergmann, Der Ultraschall und seine Anwendung in Wissenschaft und Technik, S. Hirzel Verlag, Stuttgart, 6th edn, 1954.

32 P. H. Mott, J. R. Dorgan and C. M. Roland, J. Sound Vib., 2008, 312, 572-575.

33 L. D. Landau and E. M. Lifshitz, Theory of Elasticity. Course of Theoretical Physics, Pergamon Press, Oxford, 3rd edn, 1986, vol. 7.

34 N.-S. Cheng, Ind. Eng. Chem. Res., 2008, 47, 3285-3288.

35 F. Fergusson, E. Guptill and A. MacDonald, J. Acoust. Soc. Am., 1954, 26, 67-69.

36 O. Bates, Ind. Eng. Chem., 1936, 28, 494-498.

37 F. Gucker and G. Marsh, Ind. Eng. Chem., 1948, 40, 908-915.

38 J. Dual and T. Schwarz, Lab Chip, 2012, 12, 244-252.

39 M. Wiklund, P. Spégel, S. Nilsson and H. M. Hertz, Ultrasonics, 2003, 41, 329-333.

40 J. Hultström, O. Manneberg, K. Dopf, H. M. Hertz, H. Brismar and M. Wiklund, Ultrasound Med. Biol., 2007, 33, 145-151.

41 R. Barnkob, I. Iranmanesh, M. Wiklund and H. Bruus, Lab Chip, 2012, 12, 2337-2344.

42 M. Raffel, C. E. Willert, S. T. Wereley and J. Kompenhans, Particle Image Velocimetry, Springer, New York, 2007.

43 M. Ohlin, A. Christakou, T. Frisk, B. Önfelt and M. Wiklund, Proc. 15th MicroTAS, 2 - 6 October 2011, Seattle (WA), USA, 2011, pp. $1612-1614$.

44 K. Sritharan, C. Strobl, M. Schneider, A. Wixforth and Z. Guttenberg, Appl. Phys. Lett., 2006, 88, 054102.

45 T. Frommelt, M. Kostur, M. Wenzel-Schaefer, P. Talkner, P. Haenggi and A. Wixforth, Phys. Rev. Lett., 2008, 100, 034502.

46 R. Lindken, M. Rossi, S. Grosse and J. Westerweel, Lab Chip, 2009, 9, 2551-2567.

47 C. Cierpka, M. Rossi, R. Segura and C. J. Kaehler, Meas. Sci. Technol., 2011, 22, 015401.

48 S. Sadhal, Lab Chip, 2012, 12, 2600-2611. 